OPEN

SUBJECT AREAS:

ATOMIC FORCE

MICROSCOPY

IMAGING TECHNIQUES

Received

23 September 2013

Accepted

8 November 2013

Published

27 November 2013

Correspondence and requests for materials should be addressed to

S.L. (samuele_lilliu@

hotmail.it)

\section{EFM data mapped into 2D images of tip-sample contact potential difference and capacitance second derivative}

\author{
S. Lilliu', , C. Maragliano ${ }^{2}$, M. Hampton', M. Elliott' ${ }^{1}$ M. Stefancich ${ }^{2}$, M. Chiesa ${ }^{2}$, M. S. Dahlem² \\ \& J. E. Macdonald'
} School of Physics and Astronomy, Cardiff University, Queens Buildings, The Parade, Cardiff CF243AA, United Kingdom, ${ }^{2}$ Masdar
Institute of Science and Technology, PO Box 54224, Abu Dhabi, United Arab Emirates.

We report a simple technique for mapping Electrostatic Force Microscopy (EFM) bias sweep data into 2D images. The method allows simultaneous probing, in the same scanning area, of the contact potential difference and the second derivative of the capacitance between tip and sample, along with the height information. The only required equipment consists of a microscope with lift-mode EFM capable of phase shift detection. We designate this approach as Scanning Probe Potential Electrostatic Force Microscopy (SPP-EFM). An open-source MATLAB Graphical User Interface (GUI) for images acquisition, processing and analysis has been developed. The technique is tested with Indium Tin Oxide (ITO) and with poly(3-hexylthiophene) (P3HT) nanowires for organic transistor applications.

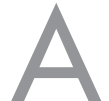

tomic Force Microscopy (AFM) has been extensively used to measure electrical properties of a sample at the nanoscale. In 1988, Y. Martin et al. ${ }^{1}$ used an AFM tip to measure the electric force between tip and sample. In this technique, established later as Electrostatic Force Microscopy (EFM), a conductive AFM tip is electrically biased against a grounded sample and the derivative of the electrostatic force is probed. EFM has been used to measure the local electrostatic properties across surfaces in a variety of applications such as thin film transistors ${ }^{2-5}$, solar cells ${ }^{6,7}$, carbon nanotubes ${ }^{8}, \mathrm{DNA}^{9}$, and surfaces in general ${ }^{10}$. Different EFM modes have been developed over the years: Scanning Capacitance Microscopy (SCM), which measures the local capacitance ${ }^{11}$; Kelvin Probe Force Microscopy (KPFM), which measures the contact potential difference $V_{C P D}$ between the tip and the sample ${ }^{12,13}$; as well as other techniques ${ }^{14-16}$. Each one of the different scanning modes is suitable for probing specific information, requiring several scans of the same area, if more than one measurement is needed. This task is often challenging since it is not straightforward to guarantee that the same area is probed after switching between different scanning modes. Considering the potential of EFM-based techniques, it would be a clear advantage if a microscope could simultaneously extract all, or at least some, of the needed information in a single scanning process. Apart from the works of Riedel et al. ${ }^{17-19}$, this challenge has been mainly addressed in AFM research using complex and costly external circuitry, such as multiple lock-in amplifiers that are able to extract information at multiple frequencies ${ }^{20}$.

The two-step EFM mode, also known as lift-mode EFM-phase, allows simultaneous reconstruction of the topography of the surface and the electrostatic force field between the tip and the sample. In the first line scan, the topography is obtained in tapping mode; in the second line scan, the electrostatic interaction is probed at larger distance while biasing the tip or the sample ${ }^{21,22}$. In lift-mode EFM-phase, the possibility of simultaneously acquiring different information about the same area of a sample represents a considerable advantage. A step forward in the direction of increasing-throughput techniques would be a method able to probe, in a single process and over the same area, different electrical parameters of the sample. These include the tip-sample contact potential difference $V_{C P D}$, which includes information on the sample work function, and the second derivative of the capacitance, which could be further processed in order to obtain a map of the sample dielectric constant ${ }^{17-19,23}$.

Recently, Riedel et al. developed a method for obtaining the local dielectric permittivity of thin films from EFMphase scans and the Equivalent Charge Method (ECM ${ }^{17-19}$. By following a similar approach, we present a technique based on lift-mode EFM-phase, which allows simultaneous probing of the second derivative of the capacitance and the contact potential difference $V_{C P D}$ between the tip and the sample, along with the surface 
topography. This information is acquired over the same scanning area, without the need of switching into a different scanning mode. We designate this approach as the Scanning Probe Potential Electrostatic Force Microscopy (SPP-EFM) technique. SPP-EFM thus allows extracting information analogous to what can be acquired with SCM and KPFM, but in a single scan. As in the case of Riedel's technique, SPP-EFM is a low-cost technique since it requires only a microscope able to operate in lift-mode EFM-phase. Contrary to other multi-parameters extraction approaches, SPP-EFM does not require additional equipment (such as external lock-in amplifiers). The method described here can be integrated with the Equivalent Charge Method (ECM $)^{17-19}$ for the quantification of the local dielectric permittivity.

The SPP-EFM technique described here is based on EFM-phase and EFM-sweep. The basics of these two techniques are here outlined.

Lift-mode EFM-phase is an AFM-based technique sensitive to the sample electrical properties. It allows imaging the electrostatic morphology of the sample on a relatively large scale (e.g. $1 \mu \mathrm{m})$. Liftmode EFM-phase consists of two steps. The surface topography is first determined by a line scan in AFM tapping mode. During this step, both the cantilever and the sample electric grounded. An EFM line scan at a certain height above the surface is then performed. The cantilever is set to electric ground and the sample is biased. The tipsample force gradient is probed by monitoring the following phase shift ${ }^{24}$ :

$$
\Delta \varphi=\left.\{\arg [\widetilde{W}(j \omega)]-\arg [W(j \omega)]\}\right|_{\omega=\omega_{n}}
$$

where $\widetilde{W}(j \omega)$ is the frequency response of the cantilever under the action of an external force field $f_{S}, W(j \omega)$ is the frequency response of the free cantilever, and $\omega_{n}$ is the cantilever natural frequency (see supporting information). To a first approximation, $\Delta \varphi$ can be written as:

$$
\Delta \varphi=-\left.\frac{Q}{K} \frac{d f_{S}(z)}{d z}\right|_{z=z_{0}}
$$

where $Q$ is the cantilever quality factor, $K$ is the cantilever elastic constant, $z$ is the tip-cantilever distance, and $z_{0}$ is the cantilever equilibrium position. Equation (2) is only valid under the following assumptions: (i) the cantilever is approximated as a monodimensional Linear Time-Invariant (LTI) system, and (ii) $K / Q \gg$ $d f_{S}(z) /\left.d z\right|_{z_{0}}$ (see supporting information).

The tip-sample electrostatic force can be written s $^{25,26}$ :

$$
f_{e l}(z)=\frac{1}{2} \frac{\partial C}{\partial z} V_{t o t}^{2}
$$

where $C=C(z)$ and $V_{\text {tot }}$ are the tip-sample capacitance and the applied bias, respectively. If tip and sample are made of different materials, the total tip-sample potential is ${ }^{12}$ :

$$
V_{t o t}=V-V_{C P D}
$$

where $V$ is an externally applied bias between tip and sample, and $V_{C P D}$ is the Contact Potential Difference $\left(V_{C P D}\right)$, i.e. the difference between the work functions of the two materials. The electrostatic force $f_{e l}(z)$ decays much more slowly as a function of the distance $z$ than the shortrange forces (e.g. Van der Waals force). If the tip-sample distance is such that the sole effect of the electrostatic force is probed by the biased tip, then the force field can be approximated as $f_{S}(z) \approx f_{e l}(z)$. Forcecurve based techniques for the exact local separation of $f_{S}(z)$ into $f_{e l}(z)$ and short-range forces have been recently proposed by Maragliano et $\mathrm{al}^{27}$. However, the implementation of these methods to obtain simultaneous 2D images of electrostatic force, short-range forces, capacitance and height, would require complex, expensive and fast analogic/ digital circuitry, able to perform force-distance curve scans at different biases, point-by-point, in the $2 \mathrm{D}$ raster scan.
By combining the previous equations, an expression for the phase shift can be obtained:

$$
\Delta \varphi=-\left.\frac{Q}{K} \frac{d f_{S}(z)}{d z}\right|_{z=z_{0}}=-\left.\frac{1}{2} \frac{Q}{K}\left[\frac{d^{2} C}{d z^{2}}\left(V-V_{C P D}\right)^{2}\right]\right|_{z=z_{0}}
$$

This expression shows that, if lift-mode EFM-phase is combined with a tip-sample DC voltage sweep, the second derivative of the capacitance and $V_{C P D}$ can be extracted with a parabolic fit. This technique ${ }^{28-30}$ can be concisely indicated as EFM-sweep. In the case of EFM-sweep, the scan size is localized to a small area of the surface, which depends on the tip-sample distance and on the tip radius (in the order of tens of nanometers $)^{27}$. With this local method, information on sample morphology is lost. Several EFM-phase data points are collected at different sample biases. The EFM-phase data is then calibrated to find $V_{C P D}$ and the capacitive contribution proportional to $d^{2} C / d z^{2}$, by using equation (5). A plot of the EFM-phase $\Delta \varphi$ versus the tip-sample applied potential $V$ produces a calibration curve that can be fitted by equation (5) and rewritten as the following parabolic expression:

$$
\Delta \varphi=a(V-b)^{2}
$$

where $a=-(Q / 2 K) \partial^{2} C / \partial z^{2}$ and $b=V_{C P D}$. Several experimental setups have been proposed to obtain the two parameters ${ }^{10,28,31-33}$. As an example, Lei et al. ${ }^{9}$ and Simmonds et al. ${ }^{34}$ evaporated gold electrodes on $\mathrm{SiO}_{2}$ and deposited polymer nanowires across them. By grounding a highly doped $\mathrm{n}^{++}$silicon tip and sweeping the bias across the electrodes from $-7 \mathrm{~V}$ and $+7 \mathrm{~V}$ along a line scan, they obtained $a$ and $b$. The calibration scan was performed in a small area, when compared to the typical AFM image size (1-10 $\mu \mathrm{m})$.

The main advantage of EFM-sweep is that it allows quantitatively and simultaneously extracting information on $a$ and $b$ parameters, on the local scale. The main disadvantage with respect to lift-mode EFM-phase is that the global sample morphology, in the large scale (e.g. $1 \mu \mathrm{m})$, cannot be visualized. Therefore, it would be valuable to develop a technique based on both EFM-sweep and lift-mode EFMphase, where full $a$ and $b$ images on a large scale are obtained. In the following sections, the SPP-EFM technique is discussed in detail.

\section{Results}

Here we show two tests performed with the SPP-EFM technique on Indium Tin Oxide (ITO) and poly(3-hexylthiophene) (P3HT) nanowires for organic electronics applications ${ }^{35-37}$.

The tested metal is a thin layer of Indium Tin Oxide deposited on a glass substrate (Buwon Act Co. Ltd, China). Figure 1 shows the following channels: a) height; b) goodness of fit $R^{2}$; c) deconvolved channel proportional to the second derivative of the tip-sample capacitance $A=(Q / 2 K) \partial^{2} C / \partial z^{2}\left[{ }^{\circ} V^{-2}\right]$; d) deconvolved channel corresponding to the tip-sample contact potential difference $V_{C P D}[V]$. The typical granular shape of ITO can be observed in the height (Figure 1a), capacitive channel A (Figure 1c), and contact potential difference channel B (Figure 1d). As shown in Figure 1b, most of the pixels of the deconvolved channels have a goodness of fit $R^{2}$ above 0.98. The terraces consisting of layers of crystalline ITO, seen in the height channel, cannot be observed in the capacitance and $V_{C P D}$ channels, which appear as a flatter surface in comparison to the height channel.

Figure 2 shows an example of a local calibration curve build from $86(V, \Delta \varphi)$ data points extracted from a single ITO grain. The fitting parabola $a(V-b)^{2}$ matches well the experimental data points, and confirms that the choice of $-5 \mathrm{~V}$ and $5 \mathrm{~V}$ as the maxima of the sweeping voltage keeps the data points within a parabolic regime. The fitted coefficient are $a=(Q / 2 K) \partial^{2} C / \partial z^{2}$ is $0.383 \pm 0.004\left[{ }^{\circ} \mathrm{V}^{-2}\right]$ and $b=V_{C P D}=0.18 \pm 0.01[\mathrm{~V}]$. Here, the standard errors correspond to the $95 \%$ confidence bounds. In Figure 2, the confidence bounds are also used to plot the $95 \%$ prediction bounds. 

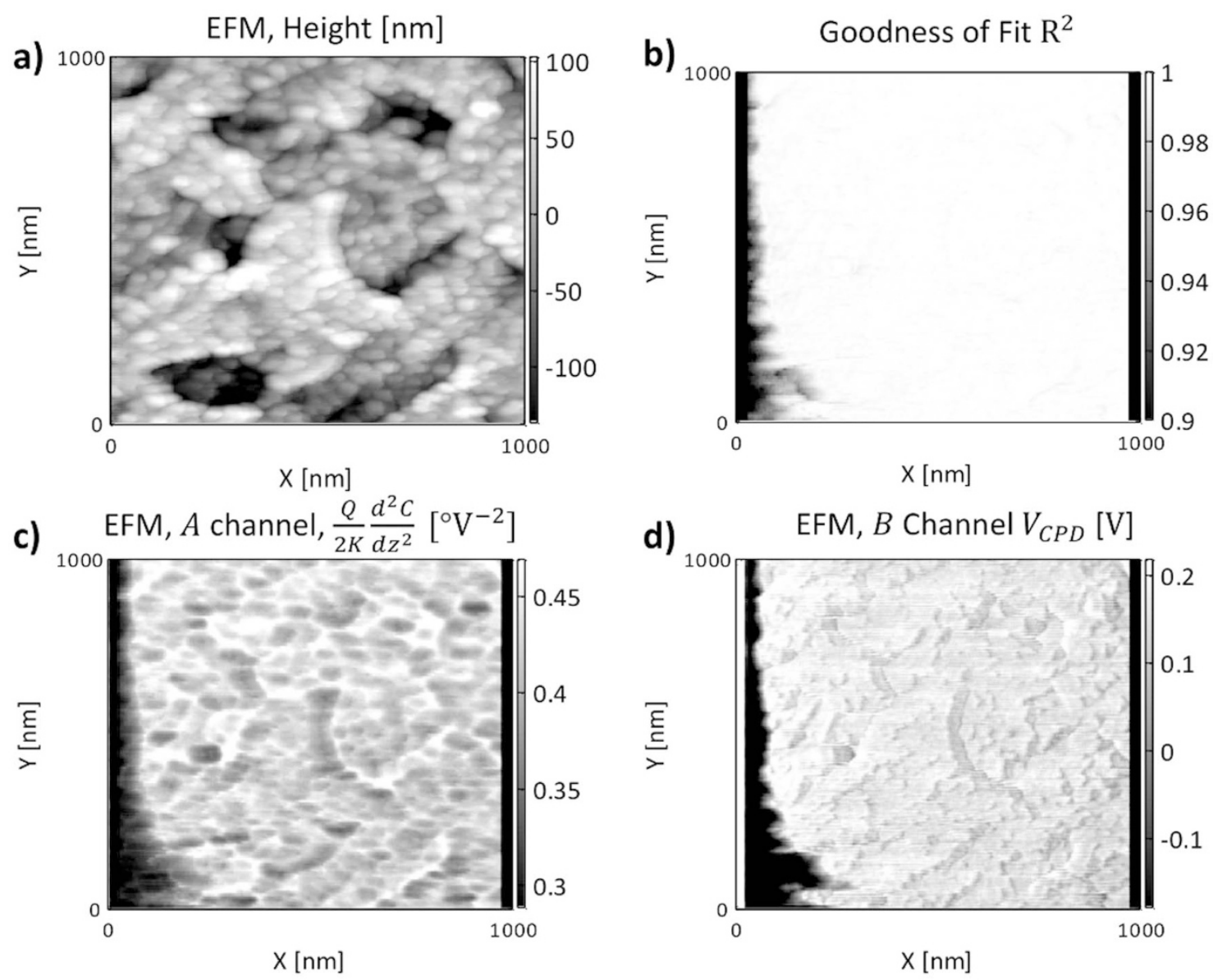

Figure 1 ITO probed by SPP-EFM. (a) Height [nm]. (b) Goodness of fit $R^{2}$. (c) Deconvolved channel proportional to the second derivative of the tipsample capacitance $(\mathrm{Q} / 2 \mathrm{~K}) \partial^{2} \mathrm{C} / \partial z^{2}\left[{ }^{\circ} V^{-2}\right]$. (d) Deconvolved channel corresponding to the tip-sample contact potential difference $V_{C P D}[\mathrm{~V}]$.

The height, the capacitance and the $V_{C P D}$ data can be compared by calculating the correlation coefficient $\rho$ between their normalized values. Height and capacitance are negatively correlated $(\rho=$ $-0.75)$. Height and $V_{C P D}$ are uncorrelated $(\rho=0.27)$, as well as capacitance and $V_{C P D}(\rho=0.06)$. This is due to the fact that the capacitive signal depends on the tip sample geometry, while the $V_{C P D}$ is independent from the sample topography in a homogeneous sample. This observation also excludes the presence of topographical artifacts in the $V_{C P D}$ signal.

Poly(3-hexylthiophene-2,5-diyl) nanowires dispersed in anisole (purity $99 \%$, Sigma-Aldrich, UK) were spincoated on a $\mathrm{Si} / \mathrm{SiO}_{2}$ substrate and annealed for 30 minutes at $140^{\circ} \mathrm{C}$. As shown in Figure 3, the wires have an average width and a thickness of $\sim 60 \mathrm{~nm}$. Figure 4 shows the following normalized channels: height, capacitance (Channel A) and $V_{C P D}$ (Channel B). The height line profile is not correlated with the capacitance $(\rho=0.02)$ and the $V_{C P D}(\rho=0.12)$ line profiles, while capacitance and $V_{C P D}$ are highly correlated $(\rho=$ $0.6)$. This is because the sample is heterogeneous (both Si substrate and nanowires are imaged simultaneously). The drift in the $V_{C P D}$, along the $y$-scan direction (top-bottom), could be an effect of tip contamination ${ }^{24}$. However, $V_{C P D}$ contrast is visible between wires and substrate because of their strong difference in work-function. Values of $V_{C P D}$ at the left borders of the nanowires are affected by high error, as shown by the goodness of fit channel. This could be due to asymmetries in the tip shape or hysteresis of the piezo, resulting in discrepancies between trace and retrace channels, in correspondence of sharp features. In the case of the piezo hysteresis, this effect could be probably avoided by performing the technique with just one trace or retrace channel, at the expense of losing in resolution and doubling the time required for a $\operatorname{scan}^{38}$.
The possibility to simultaneously map and correlate height, capacitance and $V_{C P D}$ channels could be helpful for nanowires film thickness optimization, during the development of organic transistors. Once properly calibrated with the height channel, the capacitance

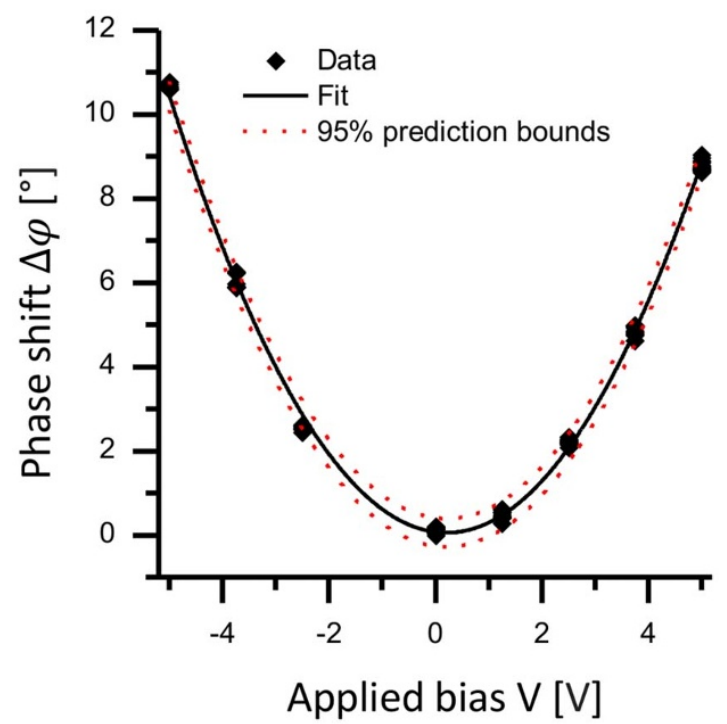

Figure $2 \mid$ Calibration parabola build from $86(V, \Delta \varphi)$ data points extracted from a homogenous region consisting of a single ITO grain. The 95\% prediction bounds are shown in the plot. Fitted values are: $a=0.383$ $\left[{ }^{\circ} V^{-2}\right]$ and $b=0.18[\mathrm{~V}]$. Standard errors based on $95 \%$ prediction bounds are: $E_{a}=0.004\left[{ }^{\circ} \mathrm{V}^{-2}\right]$ and $E_{b}=0.01[\mathrm{~V}]$. 

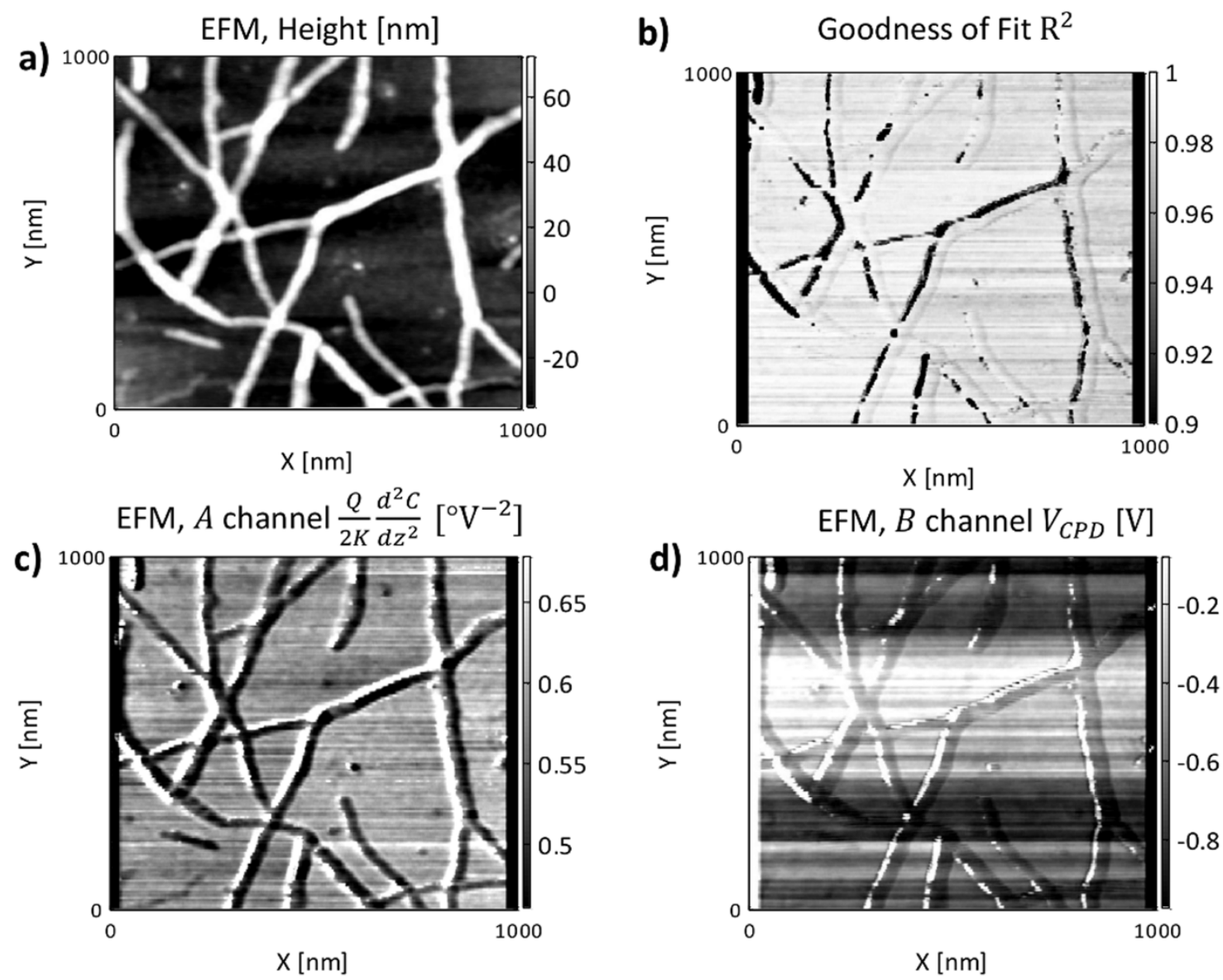

Figure 3 P3HT nanowires probed by SPP-EFM. (a) Height $[\mathrm{nm}]$. (b) Goodness of fit $R^{2}$. (c) Deconvolved channel proportional to the second derivative of the tip-sample capacitance $A=(\mathrm{Q} / 2 \mathrm{~K}) \partial^{2} C / \partial z^{2}\left[{ }^{\circ} V^{-2}\right]$. (d) Deconvolved channel corresponding to the tip-sample contact potential difference $V_{C P D}$ $[V]$.

and the $V_{C P D}$ channel could be used to measure the number of stacked nanowires in a densely packed nanowires film. Datta et al. recently demonstrated this approach in an EFM-sweep study of a Few Layers of Graphene (FLG) deposited on an insulating substrate of $\mathrm{SiO}_{2} / \mathrm{Si}^{31}$

\section{Discussion}

We have reported a technique for mapping Electrostatic Force Microscopy (EFM) sweep data into 2D images. The method allows simultaneous probing, in the same scanning area, of the contact potential difference and the second derivative of the capacitance between tip and sample, along with the height information. We designate this approach as the Scanning Probe Potential Electrostatic Force Microscopy (SPP-EFM) technique. In line with the recent challenges of simultaneous multi-information extraction, the SPP-EFM technique presents a simple solution for concurrently obtaining information similar to the one that could be recorded with two different techniques: Scanning Capacitance Microscopy (SCM) and Kelvin Probe Force Microscopy (KPFM). The SPP-EFM technique combines the advantages of lift-mode EFM-phase method, such as the superior lateral resolution when compared to lift-mode KPFM, and EFM-sweep, such as the quantitative measurements. Implemented with a microscope that can be operated in lift-mode EFM-phase, SPP-EFM is a low-cost technique that does not require any expensive extra lock-in amplifiers.

The enabling component of the SPP-EFM technique is the SPPEFM-GUI, a graphical user interface we developed under MATLAB, for image acquisition, processing and analysis. The SPP-EFM works with both Nanoscope and Asylum Research raw data files. It can also be used for basic AFM image analysis. The SPP-EFM is an opensource software, which can be freely downloaded from the supporting information (with a video tutorial and example files).

Finally, we have shown two examples of SPP-EFM analysis. Among its several applications, the SPP-EFM technique has the potential of deconvolving EFM data into contact potential images

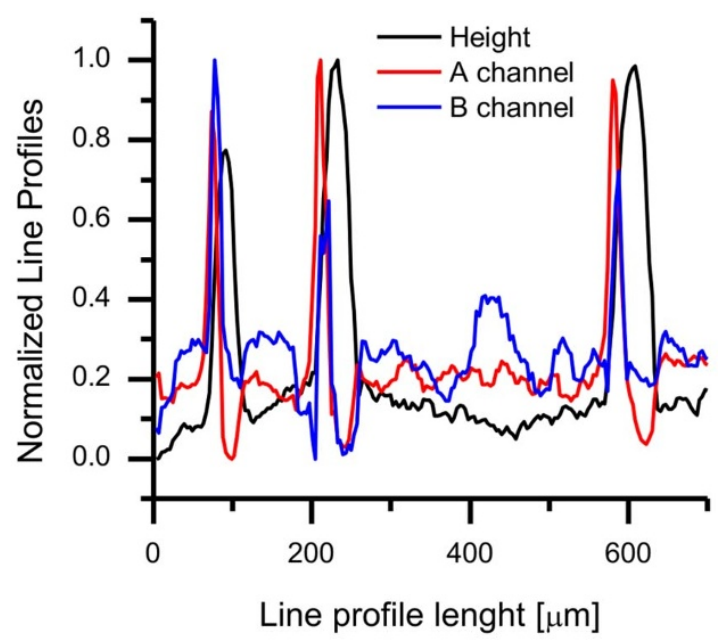

Figure $4 \mid$ Line profile across three P3HT nanowires probed by SPP-EFM, showing the following normalized channels: height, $=(Q / 2 K) \partial^{2} C / \partial z^{2}$ $\left[{ }^{\circ} \mathrm{V}^{-2}\right]$, and $B=V_{C P D}[\mathrm{~V}]$. 


\section{a) Sample biasing waveform}

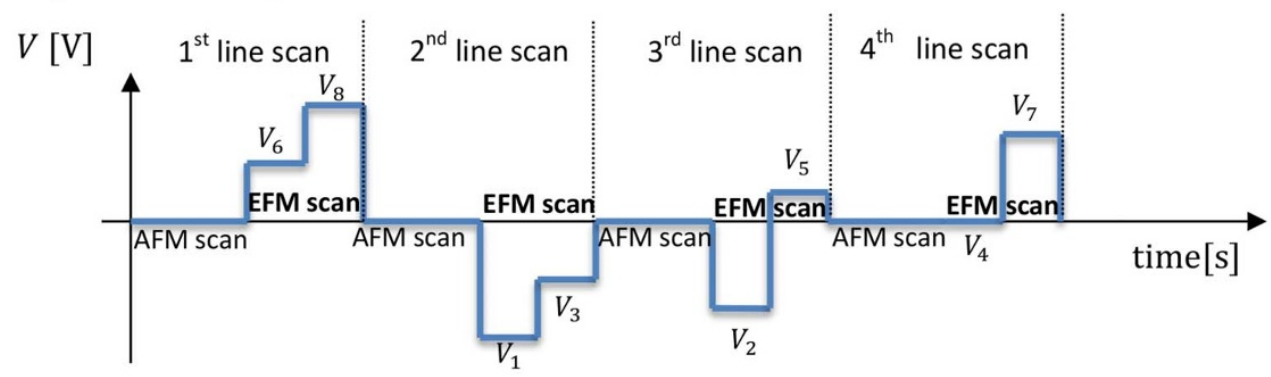

\section{b) Data used for obtaining $a_{1,1}$ and $b_{1,1}$}

\begin{tabular}{|l|l|l|l|l|}
\multicolumn{7}{|c}{$\Delta \varphi_{T}$} \\
\hline$\Delta \varphi_{T, 1,1}$ & $\Delta \varphi_{T, 1,2}$ & $\Delta \varphi_{T, 1,3}$ & $\ldots$ & $\Delta \varphi_{T, 1, M}$ \\
\hline$\Delta \varphi_{T, 2,1}$ & $\Delta \varphi_{T, 2,2}$ & $\Delta \varphi_{T, 2,3}$ & $\ldots$ & $\Delta \varphi_{T, 2, M}$ \\
\hline$\Delta \varphi_{T, 3,1}$ & $\Delta \varphi_{T, 3,2}$ & $\Delta \varphi_{T, 3,3}$ & $\ldots$ & $\Delta \varphi_{T, 3, M}$ \\
\hline$\Delta \varphi_{T, 4,1}$ & $\Delta \varphi_{T, 4,2}$ & $\Delta \varphi_{T, 4,3}$ & $\ldots$ & $\Delta \varphi_{T, 4, M}$ \\
\hline$\Delta \varphi_{T, 5,1}$ & $\Delta \varphi_{T, 5,2}$ & $\Delta \varphi_{T, 5,3}$ & $\ldots$ & $\Delta \varphi_{T, 5, M}$ \\
\hline$\ldots$ & $\ldots$ & $\ldots$ & $\ldots$ & $\ldots$ \\
\hline$\Delta \varphi_{T, M, 1}$ & $\Delta \varphi_{T, M, 2}$ & $\Delta \varphi_{T, M, 3}$ & $\ldots$ & $\Delta \varphi_{T, M, M}$ \\
\hline
\end{tabular}

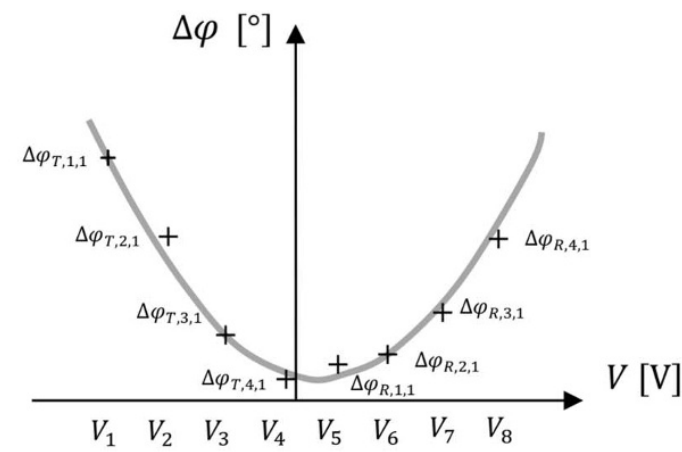

\begin{tabular}{|l|l|l|l|l|}
\hline$\Delta \varphi_{R, 1,1}$ & $\Delta \varphi_{R, 1,2}$ & $\Delta \varphi_{R, 1,3}$ & $\ldots$ & $\Delta \varphi_{R, 1, M}$ \\
\hline$\Delta \varphi_{R, 2,1}$ & $\Delta \varphi_{R, 2,2}$ & $\Delta \varphi_{R, 2,3}$ & $\ldots$ & $\Delta \varphi_{R, 2, M}$ \\
\hline$\Delta \varphi_{R, 3,1}$ & $\Delta \varphi_{R, 3,2}$ & $\Delta \varphi_{R, 3,3}$ & $\ldots$ & $\Delta \varphi_{R, 3, M}$ \\
\hline$\Delta \varphi_{R, 4,1}$ & $\Delta \varphi_{R, 4,2}$ & $\Delta \varphi_{R, 4,3}$ & $\ldots$ & $\Delta \varphi_{R, 4, M}$ \\
\hline$\Delta \varphi_{R, 5,1}$ & $\Delta \varphi_{R, 5,2}$ & $\Delta \varphi_{R, 5,3}$ & $\ldots$ & $\Delta \varphi_{R, 5, M}$ \\
\hline$\ldots$ & $\ldots$ & $\ldots$ & $\ldots$ & $\ldots$ \\
\hline$\Delta \varphi_{R, M, 1}$ & $\Delta \varphi_{R, M, 2}$ & $\Delta \varphi_{R, M, 3}$ & $\ldots$ & $\Delta \varphi_{R, M, M}$ \\
\hline
\end{tabular}

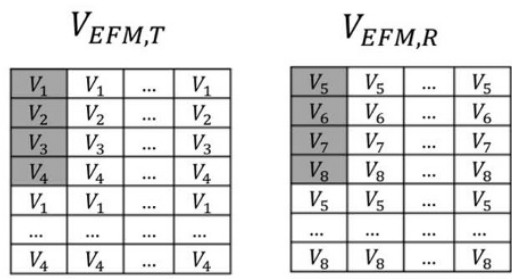

\section{c) Data used for obtaining $a_{2,1}$ and $b_{2,1}$}

\begin{tabular}{|l|l|}
\multicolumn{2}{c}{$\Delta \varphi_{T}$} \\
\hline$\Delta \varphi_{T, 1,1}$ & $\Delta \varphi_{T, 1,2}$ \\
\hline$\Delta \varphi_{T, 2,1}$ & $\Delta \varphi_{T, 2,2}$ \\
\hline$\Delta \varphi_{T, 3,1}$ & $\Delta \varphi_{T, 3,2}$ \\
\hline$\Delta \varphi_{T, 4,1}$ & $\Delta \varphi_{T, 4,2}$ \\
\hline$\Delta \varphi_{T, 5,1}$ & $\Delta \varphi_{T, 5,2}$ \\
\hline$\ldots$ & $\ldots$ \\
\hline$\Delta \varphi_{T, M, 1}$ & $\Delta \varphi_{T, M, 2}$ \\
\hline
\end{tabular}

\begin{tabular}{|l|l|}
\multicolumn{1}{c}{$\Delta \varphi_{R}$} \\
\hline$\Delta \varphi_{R, 1,1}$ & $\Delta \varphi_{R, 1,2}$ \\
\hline$\Delta \varphi_{R, 2,1}$ & $\Delta \varphi_{R, 2,2}$ \\
\hline$\Delta \varphi_{R, 3,1}$ & $\Delta \varphi_{R, 3,2}$ \\
\hline$\Delta \varphi_{R, 4,1}$ & $\Delta \varphi_{R, 4,2}$ \\
\hline$\Delta \varphi_{R, 5,1}$ & $\Delta \varphi_{R, 5,2}$ \\
\hline$\ldots$ & $\ldots$ \\
\hline$\Delta \varphi_{R, M, 1}$ & $\Delta \varphi_{R, M, 2}$ \\
\hline
\end{tabular}
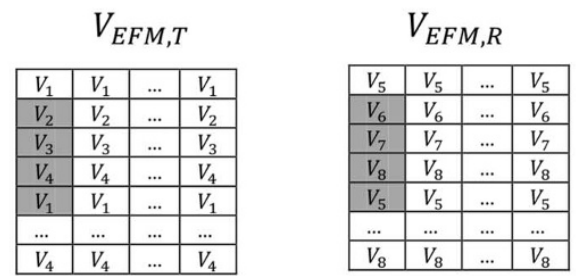

Figure $5 \mid$ Sample biasing scheme. (a) Sample biasing waveform. (b) Basic principle of SPP-EFM local calibration for the first pixels of the $A$ and $B$ channels. The 8 values from the lift-mode EFM-phase trace and retrace channels are plotted vs. the applied bias and then fitted by using the previously described parabola. (c) Generation of the pixel $(2,1)$ for $A$ and $B$ channels.

of polymer-fullerene blends for organic solar cells applications, showing a contrast between the polymer and the fullerene phase. This would not be possible with lift-mode KPFM due to its limitations in the lateral resolution. The possibility of locally discriminating between the two materials on the nano-scale would be beneficial for a better understanding of the nano-morphology of films for organic electronics applications.

\section{Methods}

SPP-EFM: system setup. Scanning Probe Potential Electrostatic Force Microscopy (SPP-EFM) measurements are performed in ambient conditions with a Nanoscope III Multimode with Extender electronics module (Bruker, USA). The Nanoscope allows recording only three channels. In order to deconvolve the lift-mode EFMphase images, represented by $\Delta \varphi$ in equation (6), into a $V_{C P D}$ image (b) and a capacitive image (a), two channels are used: EFM-phase trace and EFM-phase retrace, both in lift mode.

Commercial highly doped $\mathrm{n}^{++}$Super-Sharp tips (Nanosensors, Switzerland, nominal tip radius $2 \mathrm{~nm}$ ) are used ${ }^{9,24}$ for the scans. Before starting a scan in SPP-EFM mode, several scans in AFM mode (light tapping) are taken ${ }^{9,24}$. Usually after $\sim 3$ hours of AFM scans, the $x$ and $y$ piezo drift is minimized. Minimum drift is a necessary condition in SPP-EFM: trace and retrace line scans must be almost coincident.

As suggested by Lei ${ }^{9,24}$ and Simmonds ${ }^{34}$, we used a fixed tip-sample distance of $20 \mathrm{~nm}$ during the lift-mode scan. At this distance, lift-mode EFM-phase provides higher lateral resolution than the lift-mode $\mathrm{KPFM}^{9,24}$, which directly probes $V_{C P D}$.

SPP-EFM: sample biasing waveform. The biasing waveform can either be applied via the AFM control software or with an external waveform generator. A line scan in lift-mode EFM-phase is made of 4 contributions: AFM trace and retrace, EFM trace and retrace. The tip is always grounded, and a voltage waveform is applied to the sample. During the AFM trace and retrace the bias applied to the sample is zero, while 
during the lift-mode non-zero biases are applied. Several waveform combinations have been tested. When linear voltage sweeps ${ }^{9,24,34}$ are used in a single line (for example a saw tooth waveform), the sensed bias cannot follow exactly the applied bias, probably due to capacitive effects. In order to avoid these problems, a varying bias during the lift-mode scan should be avoided. The best results have been obtained by maintaining a constant voltage in each EFM line scan. The optimized sample biasing waveform used in the present work is shown in Figure 5a. The trace raster scan is made of $512 \times 512$ pixels. Each raster point corresponds to one element of the biasing matrix $V_{E F M, T}$, shown in Figure $5 \mathrm{~b}$. Each column of $V_{E F M, T}$ is divided into the

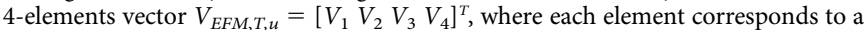
different applied bias. This vector repeats along the column as shown in Figure $5 b-c$. This biasing scheme is then repeated for all columns, in order to have a constant voltage along each EFM line scan. At each raster scan point, $\Delta \varphi$ is measured. This gives a phase difference matrix indicated as $\Delta \varphi_{T}$. An analogous biasing scheme is employed for the retrace raster scan. However, in this case, a different 4-elements biasing vector $V_{E F M, T, u}=\left[V_{5} V_{6} V_{7} V_{8}\right]^{T}$ is applied. We refer to the biasing matrix as $V_{E F M, T, R}$, and to the measured phase difference matrix as $\Delta \varphi_{R}$ (Figure $1 \mathrm{~b}-\mathrm{c}$ ).

In most of the scans, an AFM scan rate of $1 \mathrm{~Hz}$ is used. The waveform maximum amplitude is set to $\pm 5 \mathrm{~V}$, in order to have a nearly perfect parabolic relationship. Larger biases result in strong deviations of $\Delta \varphi$. The EFM channel parameters in the Nanoscope software must be set in a way that no automatic flattening is applied to the acquired EFM-phase images ${ }^{34}$.

SPP-EFM: software design and data processing. Once the scan has been completed, height, lift-mode EFM-phase trace and retrace channels are automatically saved in a Nanoscope raw data file by the Nanoscope software. An open-source graphical user interface software (SPP-EFM GUI) has been developed with MATLAB for SPP-EFM data acquisition and processing, and is freely available, with a video tutorial and example files, in the supporting information. The software was initially developed for processing Nanoscope binary files, but is currently also able to process Asylum Research binary files.

Multiple Nanoscope binary raw data files can be imported and converted into height and EFM-phase data and visualized in the software. Information on the scan (header) is automatically imported from the binary file by using regular expressions. Workflow sessions can be saved and reloaded. Images can be zoomed, panned, contrast-adjusted, and saved in a ready-to-publish vectorial file format. Multiple line profiles from all the channels can be extracted along a line and plotted.

The objective of the fitting routine in the SPP-EFM technique is to deconvolve the two EFM-phase images into 3 main extra channels (2D images): $A$, the capacitive contribution (from the $a$ parameters in equation (6)); $B$, the $V_{C P D}$ contribution (from the $b$ parameter in equation (6)); and $R^{2}$, the goodness-of-fit. Further channels representing the fitting error on the $a$ and $b$ parameters are also available. The deconvolution scheme for the first pixel $(1,1)$ of the 3 new channels is descibed in Figure $5 \mathrm{~b}$. The deconvolution scheme for the pixel $(2,1)$ of the 3 new channels is described in Figure 5c. In general, the $(i, j)$-th pixel of the $A$ and $B$ channels, where $i$ and $j$ are respectively the row and column indexes, is obtained by fitting the following $(V, \Delta \varphi)$ points:

$$
\begin{aligned}
& V=\left[V_{E F M, T}(i: i+3, j) V_{E F M, R}(i: i+3, j)\right] \\
& \Delta \varphi=\left[\Delta \varphi_{T}(i: i+3, j) \Delta \varphi_{R}(i: i+3, j)\right]
\end{aligned}
$$

where $j$ is fixed and $i: i+3$ indicates the following rows: $i, i+1, i+2, i+3$. Each step involves a parabolic fitting with the MATLAB function fit. It is clear from equation (7), that the fitting scheme consists of a 'moving average' along the columns, with an averaging window of 4 pixels. Therefore some of the EFM lateral resolution along the $y$-scan direction is lost. However, no information is lost along the $x$-scan direction. The resolution loss along the $y$-direction could be improved (on a different AFM system) by simply increasing the number of pixels per image, at the price of a lower scan speed (and a higher drift). Further details about the fitting scheme are reported in the supporting information. Since images are acquired with a resolution of $512 \times 512$ pixels, the number of fitting steps is equal to 262,144 . Parallel computing is used to speed up the computation. Multiple images can be batch deconvolved.

After the deconvolution is completed, 3 new extra main channels appear: $A=(Q /$ $2 K) \partial^{2} C / \partial z^{2}, B=V_{C P D}$, and $R^{2}$. Secondary channels displaying the fit error on $A$ and $B$ are also available. Local calibration curves can be built by drawing arbitrary shapes around features of interest in any channel. In this way, the average $A$ and $B$ parameters related to that region are extracted. Statistics about each single channel can be extracted by selecting data resulting only from a good fit, i.e. by using the $R^{2}$ information. A $R^{2}$ threshold value of 0.99 , for example, extracts only the pixels with $R^{2}$ $>0.99$, and creates an image mask which is applied to the desired channel to extract statistic information. Using the created mask, the histogram of the current channel can be extracted and displayed in the edit box. This procedure can be batch applied to multiple files.

Finally a significant improvement of our method could be achieved by integrating it with the ECM technique proposed by Riedel et al..$^{21-23}$ for the conversion of the $A=$ $(Q / 2 K) \partial^{2} C / \partial z^{2}$ channel into a map of the dielectric permittivity.

All the mathematical steps, from the basic mass-spring-damper (representing the cantilever dynamics) to the expression of the phase shift $\Delta \varphi$ probed in EFM mode, are shown in a rigorous and simple way in the supporting information. The SPP-EFMGUI, the graphical user interface we developed under MATLAB, for image acquisition, processing and analysis, can be downloaded from The software can be downloaded from: http://www.lilliu.co.uk/toolboxes/SPM/SPC-EFM_GUI.zip. A video tutorial on the SPP-EFM-GUI is available at the following link: http://www.youtube. com/watch?v=yQWIAyBhFO0.

1. Martin, Y. et al. High-resolution capacitance measurement and potentiometry by force microscopy. Appl. Phys. Lett. 52, 1103-1105 (1988).

2. Bürgi, L. et al. A microscopic view of charge transport in polymer transistors. Synthetic Met. 146, 297-309 (2004).

3. Bürgi, L. et al. Close look at charge carrier injection in polymer field-effect transistors. J. Appl. Phys. 94, 6129-6137 (2003).

4. Bürgi, L. et al. Noncontact potentiometry of polymer field-effect transistors. Appl. Phys. Lett. 80, 2913-2915 (2002).

5. Ong, B. S. Design of high-performance regioregular polythiophenes for organic thin-film transistors. P. IEEE 93, 1412 (2005).

6. Coffey, D. C. et al. Time-resolved electrostatic force microscopy of polymer solar cells. Nat. Mater. 5, 735-740 (2006).

7. Visoly-Fisher, I. et al. How Polycrystalline Devices Can Outperform SingleCrystal Ones: Thin Film CdTe/CdS Solar Cells. Adv. Mater. 16, 880 (2004).

8. Bachtold, A. et al. Scanned Probe Microscopy of Electronic Transport in Carbon Nanotubes. Phys. Rev. Lett. 84, 6082 (2000).

9. Lei, C. H. et al. Conductivity of macromolecular networks measured by electrostatic force microscopy. Appl. Phys. Lett. 83, 482-484 (2003).

10. Lu, Y. et al. Electrostatic Force Microscopy on Oriented Graphite Surfaces: Coexistence of Insulating and Conducting Behaviors. Phys. Rev. Lett. 97 (2006).

11. Williams, C. C. et al. Lateral dopant profiling with $200 \mathrm{~nm}$ resolution by scanning capacitance microscopy. Appl. Phys. Lett. 55, 1662-1664 (1989).

12. Nonnenmacher, M. et al. Kelvin probe force microscopy. Appl. Phys. Lett. 58, 2921-2923 (1991).

13. Girard, P. Electrostatic force microscopy: principles and some applications to semiconductors. Nanotechnology 12, 485 (2001).

14. Aoki, N. et al. Scanning gate microscopy of copper phthalocyanine field effect transistors. Appl. Phys. Lett. 91, 192113 (2007).

15. Saurenbach, F. et al. Imaging of ferroelectric domain walls by force microscopy Appl. Phys. Lett. 56, 1703-1705 (1990).

16. Kalinin, S. V. et al. Local potential and polarization screening on ferroelectric surfaces. Phys. Rev. B 63, 125411 (2001).

17. García, R. et al. Dynamic atomic force microscopy methods. Surf. Sci. Rep. 47, 197-301 (2002)

18. Elings, V. B. et al. Scanning probe microscope using stored data for vertical probe positioning. (1994)

19. Jacobs, H. et al. Surface potential mapping: A qualitative material contrast in SPM. Ultramicroscopy 69, 39-49 (1997).

20. Fumagalli, L. et al. Dielectric-constant measurement of thin insulating films at low frequency by nanoscale capacitance microscopy. Appl. Phys. Lett. 91, 243110 (2007).

21. Riedel, C. et al. Nanoscale dielectric properties of insulating thin films: From single point measurements to quantitative images. Ultramicroscopy 110, 634-638 (2010).

22. Riedel, C. et al. Determination of the nanoscale dielectric constant by means of a double pass method using electrostatic force microscopy. J. Appl. Phys. 106, 024315 (2009).

23. Riedel, C. et al. Nanodielectric mapping of a model polystyrene-poly(vinyl acetate) blend by electrostatic force microscopy. Physical Review E 81, 010801 (2010).

24. Lei, C. H. et al. Quantitative electrostatic force microscopy-phase measurements. Nanotechnology 15, 627 (2004).

25. Paris, D. T. et al. Basic Electromagnetic Theory. (McGraw Hill, 1969).

26. Feynman, R. P. et al. The Feynman Lectures on Physics. (Addison-Wesley Publishing Company Inc., 2011).

27. Maragliano, C. et al. Dynamic electrostatic force microscopy technique for the study of electrical properties with improved spatial resolution. Nanotechnology 24, 225703 (2013).

28. Gekhtman, D. et al. Electrostatic Force Spectroscopy and Imaging of Bi Wires: Spatially Resolved Quantum Confinement. Phys. Rev. Lett. 82, 3887 (1999).

29. Burnett, T. et al. Mapping of Local Electrical Properties in Epitaxial Graphene Using Electrostatic Force Microscopy. Nano Lett. 11, 2324-2328 (2011).

30. Aykutlu, D. et al. Electrostatic force spectroscopy of near surface localized states. Nanotechnology 16, S125 (2005).

31. Datta, S. S. et al. Surface Potentials and Layer Charge Distributions in Few-Layer Graphene Films. Nano Lett. 9, 7-11 (2008).

32. Cockins, L. et al. Spatially resolved low-frequency noise measured by atomic force microscopy. Phys. Rev. B 79, 121309 (2009).

33. Piarristeguy, A. A. et al. High resolution electrical characterisation of Agconducting heterogeneous chalcogenide glasses. Solid State Ionics 181, 1205-1208 (2010).

34. Simmonds, H. et al. Self-assembled organic nanowires: A structural and electronic study. J. Vac. Sci. Technol. B 27, 831-835 (2009).

35. Lilliu, S. et al. Inkjet-printed organic photodiodes. Thin Solid Films 520, 610-615 (2011).

36. Lilliu, S. et al. The Influence of Substrate and Top Electrode on the Crystallization Dynamics of P3HT:PCBM Blends. Energy Procedia 31, 60-68 (2012). 
37. Lilliu, S. et al. Effects of Thermal Annealing Upon the Nanomorphology of Poly(3hexylselenophene)-PCBM Blends. Macromol. Rapid Commun. 32, 1454-1460 (2011).

38. Ziegler, D. et al. Improved accuracy and speed in scanning probe microscopy by image reconstruction from non-gridded position sensor data. Nanotechnology $\mathbf{2 4}$, 335703 (2013)

\section{Acknowledgments}

This work was partially funded by the Engineering and Physical Sciences Research Council (EPSRC, grant number EP/F016255), granted for the UKOPV collaboration (Cardiff University, Sheffield University, Cambridge University, Diamond Light Source).

\section{Author contributions}

S.L. and C.M. developed the technique, carried on the experiments, prepared the figures, and wrote the main manuscript. M.H. prepared the P3HT nanowires samples. J.E.M. and
M.S.D. supervised the project. M.S., M.C. and M.E. helped with the theoretical development of the technique.

\section{Additional information}

Supplementary information accompanies this paper at http://www.nature.com/ scientificreports

Competing financial interests: The authors declare no competing financial interests. How to cite this article: Lilliu, S. et al. EFM data mapped into 2D images of tip-sample contact potential difference and capacitance second derivative. Sci. Rep. 3, 3352; DOI:10.1038/srep03352 (2013).

(c) (i) $(-)$ This work is licensed under a Creative Commons AttributionBy NG No NonCommercial-NoDerivs 3.0 Unported license. To view a copy of this license, visit http://creativecommons.org/licenses/by-nc-nd/3.0 